\title{
Towards Approximate Fair Bandwidth Sharing via Dynamic Priority Queuing
}

\author{
Carmelo Cascone $e^{*}$, Nicola Bonelli ${ }^{\dagger}$, Luca Bianchi*, Antonio Capone*, Brunilde Sansò ${ }^{\ddagger}$ \\ * Politecnico di Milano, Italy \\ \{carmelo.cascone, antonio.capone\}@polimi.it, luca14.bianchi@mail.polimi.it \\ $\dagger$ Università di Pisa, Italy \\ nicola.bonelli@for.unipi.it \\ \$ Ecole Polytechnique de Montréal, Canada \\ brunilde.sanso@polymtl.ca
}

\begin{abstract}
We tackle the problem of a network switch enforcing fair bandwidth sharing of the same link among many TCPlike senders. Most of the mechanisms to solve this problem are based on complex scheduling algorithms, whose feasibility becomes very expensive with today's line rate requirements, i.e. 10-100 Gbit/s per port. We propose a new scheme called FDPA in which we do not modify the scheduler, but instead we use an array of rate estimators to dynamically assign traffic flows to an existing strict priority scheduler serving only few queues. FDPA is inspired by recent advances in programmable stateful data planes. We propose a design that uses primitives common in data plane abstractions such as P4 and OpenFlow. We conducted experiments on a physical $10 \mathrm{Gbit} / \mathrm{s}$ testbed, we present preliminary results showing that FDPA produces fairness comparable to approaches based on scheduling.
\end{abstract}

\section{INTRODUCTION}

It has been reported that TCP traffic represents $80-90 \%$ of the packets and bytes flowing today through the Internet [6]. It follows that most of the traffic sources adapt their sending rate according to the perceived available bandwidth. Indeed, TCP is the instantiation of an important design choice that contributed to the success of the Internet: to leave congestion control to the end-systems, thus permitting a relatively simpler implementation of the interconnection devices. TCP rate control algorithms, such as Additive-Increase-MultiplicativeDecrease (AIMD), help maintain a fair allocation of network resources on a per-flow basis. In the simplest case of multiple TCP streams, all experiencing the same RTT and sharing the same FIFO queue, each flow tends to occupy the same portion of the link bandwidth [19].

However, relying only on end-systems to guarantee fairness is not enough due to ill-behaving users and issues intrinsic to TCP-like algorithms. Examples of cases of unfairness are: (i) applications that open a large number of parallel TCP connections, e.g. peer-to-peer, or that tweak TCP to get better performances; (ii) non-TCP-like protocols, i.e. protocols that do not respond to congestion signals such as drops, and (iii) the dependence of standard TCP to the round-trip times (RTT) [19].

For these reasons, most Internet service providers (ISPs) tend throttle customer traffic at the network edge, limiting

978-1-5386-0728-2/17/\$31.00 (c)2017 IEEE the maximum bandwidth of each user to a feasible, but static network allocation. This approach allows ISPs to leave their core and interconnections with other ISPs uncongested at all the time. The downside is that the excess bandwidth remains unused, even in common situations of low usage, such at night.

Researchers have proposed solutions to enforce a more dynamic bandwidth allocation in the network interconnection devices. In these approaches, instead of capping the maximum sending rate at all times, network devices are able to redistribute the unused capacity (if any) to those users asking for more. The trick here is to design a bandwidth enforcement scheme that (i) guarantees that all users can obtain at least the level of service they paid for, i.e. minimum rate guarantees, and (ii) when unused capacity is available, that is shared by all users, with no one prevailing on others. Ideally, such mechanism should be introduced in the network without compromising today's line rate requirements, i.e. 10$100 \mathrm{Gbit} / \mathrm{s}$ per port.

Fair Queuing (FQ) scheduling [9], [16] is the textbook approach to enforce almost perfect fairness among different traffic sources, independently of the behavior of the endhosts. A switch implementing FQ works by assigning users to different queues, where a "user" is an arbitrary aggregate of packets, e.g. with the same IP source address or the same TCP/UDP 5-tuple. FQ provides high precision of bandwidth partitioning, but unfortunately, such precision comes at a considerable expense: (i) the time to process a packet depends on the number $N$ of active users, precisely $O(\log (N))$; and (ii) $N$ per-user queues are required.

The first limitation is important with today's throughput requirements which drastically reduce the maximum processing time allowed for a packet, e.g. a switching chip with aggregate throughput of $1 \mathrm{~Tb} / \mathrm{s}$ has a time budget of $1 \mathrm{~ns}$ to process a minimum size packet. The second limitation affects switching hardware implementations. Here the number of queues impacts both the memory requirements and the combinatorial logic necessary to implement the scheduler circuitry. Indeed, for a scheduler to be work-conserving, i.e. to serve a packet if at least one can be served, all $N$ queues must be examined at the same time. Thus, the number of wires to implement such a structure depends on $N$. As a consequence, it is hard to scale 
FQ implementations to hundreds, thousands or more users. For this reason the number of queues available in commercial hardware switches is usually bounded to less than 10 [1]. This consideration is also at the base of legacy quality of service (QoS) approaches such as DiffServ, where traffic is aggregated into few classes.

In this work, our focus is to devise a design for a bandwidth enforcing scheme in which both time and implementation complexity do not depend on the number of active users $N$.

This work is inspired by recent advances in SoftwareDefined Networking (SDN) and data plane programmability. Emerging abstractions such as P4 [5], OpenState [2], OPP [3], FAST [15], and Domino [22] allow network operators to perform flexible stateful packet processing inside the network. The statefulness of the aforementioned approaches, lays in the ability to program forwarding rules that read and modify data plane's forwarding state. Based on this capability, a number of studies have been published, showing how to implement existing and new forwarding functions using programmable data planes [8], [12], [20], [24].

We follow this path and design a scheme to enforce fair bandwidth sharing that is amenable with programmable data plane abstractions. To this purpose we do not modify the scheduler, we use instead a widely-deployed strict priority scheduler with only few queues. Fairness is enforced by dynamically assigning priorities to users according to their sending rate history. We call our design FDPA (Fair Dynamic Priority Assignment). In FDPA, packets belonging to a user whose arrival bitrate is equal or less than its fair share are given priority over those users generating traffic at higher rates. FDPA does not provide precise bit-level or packet-level fairness, but it approximates a fair repartitioning over longer timescales, in the order of few RTTs.

The scalability of FDPA does not depend on the number of queues, but instead on the state available for the rate estimator. Precisely, while the circuitry to implement a rate estimator can be shared among many flows, the switch is required to maintain per-user state, i.e. the measured rate. Hence, the only limit of FDPA is the memory available in a switching chip.

In this work we address the applicability of the FDPA approach by performing experiments on a $10 \mathrm{Gbit} / \mathrm{s}$ testbed using a software prototype implementation. Results show that FDPA produces fairness comparable to other schemes based on scheduling. However, we find that FDPA introduces a tradeoff between fairness and throughput, in which one or the other are penalized.

To summarize, the contributions of this paper are:

- Design of FDPA, a scheme to enforce approximate fair bandwidth sharing among many users. Switch requirements to support FDPA are a (i) strict priority scheduler and (ii) the ability to manage data plane's state to measure the arrival bitrate of each user.

- Evaluation of FDPA and other Linux's traffic management schemes using a $10 \mathrm{Gbit} / \mathrm{s}$ testbed with real TCP traffic.
We begin by reviewing the related work in Section II, we then introduce the FDPA design in Section III and discuss its implementation options with programmable data planes. In Section IV we present the experimental results from the 10 Gbit/s testbed, before concluding with a discussion on open questions and future work in Section V.

\section{RELATED WORK}

To reduce implementation and time complexity of FQ, a number of algorithms have been proposed in the literature. Deficit Round Robin (DRR) [21] is probably the most known and widely-deployed one. DRR was proposed to address the time complexity of FQ. Indeed, DRR achieves $O(1)$ execution time per packet. However, DRR still requires per-user queues, greatly limiting the maximum number of distinct users that can be served by the scheduler.

To overcome DRR's limitations, further approximations have been proposed. Stochastic Fair Queuing (SFQ) [13] is a probabilistic variant of FQ. Here traffic streams are hashed onto a smaller number of queues, and the hash function is periodically perturbed to minimize the time where 2 users collide onto the same queue. Here the quality of the approximations depends on the number of queues, and the perturbation interval. Finally, Approximate Fair Dropping (AFD) [17] employs a form of active queue management (AQM) by dropping packets before being stored on a simple FIFO queue. Dropping decisions are based on the recent history of packet arrivals, with higher probability of drop for users sending at higher rates. AFD has been used in several switch and router platforms at Cisco Systems [18].

Our approach shares the same design principles of AFD: (i) avoid using per-user queues in favor of per-user soft state, and (ii) achieve bandwidth partitioning by opportunistically dropping or delaying packets rather than enforcing rate by using scheduling. However, while the AFD design allows for an efficient implementation in a fixed-function ASIC, its realization with programmable data plane primitives might not be straightforward. Specifically, AFD requires the implementation of a shadow buffer in which packets are removed at random. We are not aware of any data plane abstraction providing native support for such data structure. Its behavior could be approximated using other primitives, however this would require a dedicated study. Instead, we prefer to explore the feasibility of FDPA which, as will be discussed in Section III-B, requires much simpler primitives exposed already by current data plane abstractions.

Finally, a more recent approach named PIFO has been proposed to address the need of a programmable scheduler [23]. However, similarly to fixed-function schedulers, in PIFO the number of distinct flows that can be served with a fair queuing discipline is bounded by the number of queues. In their proposed design, such bound is 2048 in total or 32 per port in a 64 port switch. While one could imagine dedicating all 2048 queues to a port, the authors do not provide any evaluation of their scheduler with realistic traffic traces. 


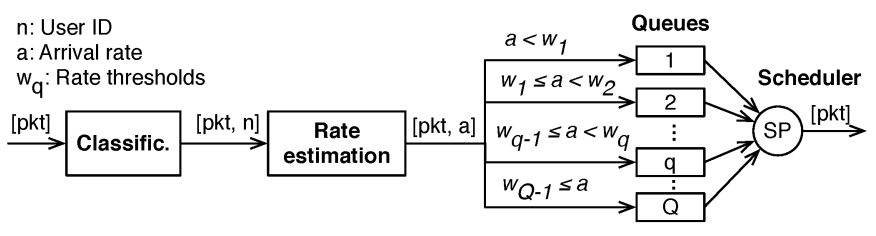

Fig. 1. FDPA forwarding pipeline.

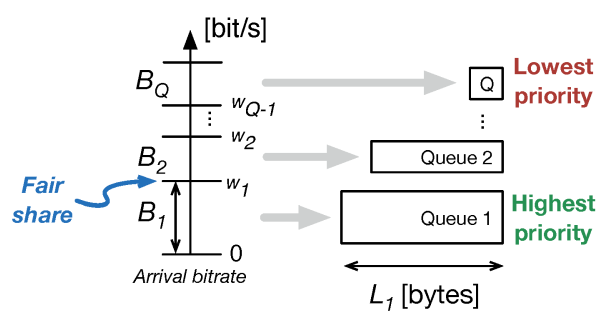

Fig. 2. Rate bands and queue size in FDPA

\section{FDPA DESIGN}

In this section we describe the design of a packet forwarding pipeline implementing FDPA. To simplify the exposition and without loss of generality, we assume a switch with rate controlled only on one egress port.

Figure 1 depicts the design of the pipeline. Packets are first classified per user and then processed by a rate estimator which measures the arrival bitrate of the specific user. Packets are then stored in one of the $Q$ priority queues such that the higher is the arrival rate, the lower will be the priority. A strict priority scheduler (SP) serves queues in priority order: packets of priority $q$ are dequeued only if all other queues with higher priority are empty, where $q=1$ is the highest priority.

The measured arrival rate for a given user at a given point in time, determines an active band for that user. Packets arrived in band $B_{q}$ will be assigned with priority $q$ (Figure 2). The first band $B_{1}$ represents the minimum guaranteed portion of the link capacity allocated to each user, for this reason $B_{1}$ should be dimensioned such that $N \times B_{1} \leq$ LinkCapacity. Moreover, to further penalize ill-behaving users, each queue has a different size $L_{q}$, with smaller values for low priority queues.

\section{A. Rationale}

To discuss the rationale behind this design, we begin with the case of a scheduler with only 2 queues $(Q=2)$, high priority and low priority; we then explain the need for more queues.

Two priorities. When congestion occurs, users sending below their fair share are prioritized against others sending at higher rates. Packets with low priority are delayed and in the worst case of a full buffer, dropped upon arrival. Such an event signals the TCP source to reduce the transmit rate. With FDPA, this reduction is expected to continue until the transmit rate hits the first band, in which case the user is prioritized again. Assuming that all sources are TCP-like and produce long-lived flows, under severe congestion we expect traffic sources to shape their transmit rate around their fair share, i.e. the upper threshold of $B_{1}$.

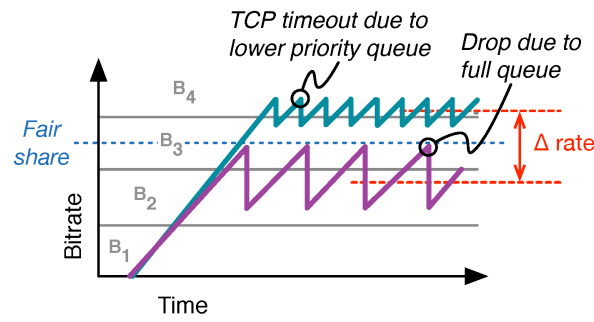

Fig. 3. Example of 2 TCP sources competing for the excess bandwidth when using more than 2 priorities.

Unfortunately, swapping queues frequently can cause packet reordering at the receiver, confusing TCP congestion control and affecting throughput. The problematic part is when users are prioritized again, i.e. their assigned queue is changed to the one with high priority. Here the same burst of consecutive packets might be stored first in the low priority queue and then in the high priority one, with the effect of having subsequent packets being transmitted before those arrived earlier. We are interested in measuring this effect when using FDPA.

In the case of non-elastic sources, e.g. constant bitrate, $B_{1}$ represents the maximum rate that a source can send with guarantees of bounded latency and minimum drop probability. Indeed when a user hits the first band, packets are always served by the same, maximum priority queue, hence preventing disruption from other TCP sources aiming to transmit at higher rates.

However, if some sources are using less than their fair share or because not all the link capacity has been reserved, i.e. $N \times B_{1}<$ LinkCapacity, using only two priorities does not enforce equal distribution of the excess bandwidth. Indeed, if we assume that capacity has been allocated for many users, but only few of them are active and sending TCP traffic, we can expect that those users will be competing in the same low priority FIFO queue, without any guarantee of fairness.

More priorities. To enforce equal distribution of the excess bandwidth, we need to introduce more priorities, such that the more a source increase its sending rate, the lower will be the priority compared to other users. When all sources are TCPlike, following the same rationale of the previous case, we expect the transmit rate of each user to converge to a fair share that considers the excess bandwidth. Such fair share will lay in a rate band other than $B_{1}$

Figure 3 illustrates the expected behavior of 2 TCP-like sources competing for the excess bandwidth. In this example, one source (1) is ill-behaving as it uses a more aggressive rate control algorithm (similar to the case of a user opening multiple TCP streams); the other source (2) is well behaving, as for each congestion signal it halves its transmit rate. At steady state, both sources tend to share the same queue with priority 3 , however the different rate-control behavior that they implement causes them to oscillate around different average values. Indeed, (1) always tends to increase its rate until it falls in the 4th band, which cause its packets to timeout as the scheduler will spend as much time as needed to serve packets of higher priority; (2) instead has higher drop probability when 


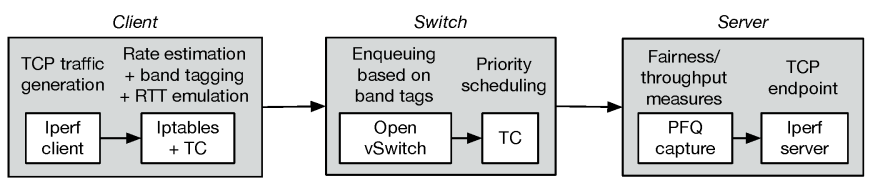

Fig. 4. Software-based processing pipeline used in experiments.

it falls in band $B_{3}$, as here the queue is monopolized by packets of (1). However, by always assuring an higher priority for lower rates, increase of (2) are always guaranteed at least until the lower threshold of band $B_{3}$. Intuitively, we expect that the difference between the average transmit rate $(\Delta$ rate $)$ will be smaller with narrower bands, hence producing a more fair allocation.

Unfortunately, as in the case with only 2 priorities, we expect that multiple narrower bands will increase the risk of packet reordering, affecting the overall throughput. We are interested in measuring such a trade-off between fairness and throughput.

\section{B. Implementation with programmable data planes}

Classifying packets per user is easy and can be done using a match-action table as defined by OpenFlow [14] or P4 [5]. Using such tables one can match on specific header fields and write the corresponding user ID $n$ on the packet's metadata.

Estimating the bitrate of a flow might be tricky at line rate. In the simplest case, the switch needs to maintain for each user a byte counter and a timestamp of the last time the rate estimation was updated. Updates of the rate values are triggered by packets arrival if the timestamp of the packet exceeds a predefined interval, i.e. the minimum interval over which the average bitrate is evaluated. The rate is then computed dividing the number of bytes by the interval between the packet's timestamp and the stored timestamp. While division is an operation that might be hard to perform in a line rate switch, in [20] it is shown how this operation can be approximated with good precision using lookup tables available in programmable data planes. A second match-action table can then be used to direct packets to the different queues according to the estimated rate band, written in the packet's metadata.

Along with programmable data planes, FDPA can be implemented in switches supporting OpenFlow v1.3+. Indeed, OpenFlow define "meters" that can be configured with different bands as defined by FDPA, such that packets hitting a given rate can be marked using the DSCP field.

Finally, priority schedulers are a common component available in today's switching hardware.

\section{EXPERIMENTAL RESULTS}

We now evaluate the feasibility and performance of FDPA using a software-based prototype implementation. We are interested in measuring the effects of different band assignment on both fairness and throughput. We also compare FDPA with other approaches such as DRR.

\section{A. Testbed}

We used 3 desktop machines with 8-core Intel Xeon E51660V3 CPUs $(3.0 \mathrm{GHz})$, equipped with multiple Intel 82599 10GbE NICs. One machine acts as a switch with 4 $10 \mathrm{Gbits} / \mathrm{s}$ ports, another machine is used to generate traffic from 2 ports, while the last is used to both generate and receive traffic from different ports. Each machine runs a Debian 9.0 Stretch based on a Linux Kernel v4.9.16.

Figure 4 shows the processing pipeline used to emulate FDPA. We use iperf to generate TCP traffic, Linux's iptables to estimate the rate and tag packets accordingly. In our design, rate estimation should happen in the switch, however, to simplify the prototype implementation we decided to move it to the client machines. We use Linux's tc (Traffic Control) to emulate different RTTs at the clients and to perform priority scheduling at the switch. Open vSwitch is used to steer packets to the different queues based on the band tags. Finally, we use PFQ [4], a framework for accelerated packet I/O, to measure the bitrate of each user. Both clients and server use TCP Cubic, with the default parameters found in the Linux Kernel v4.9.16. We only adjust the memory available to TCP buffers to allow for a large number of connections. We set the MTU of all interfaces to 1500 bytes.

We configure sources to experience an emulated RTT of around $5 \mathrm{~ms}$ with maximum $0.25 \mathrm{~ms}$ of variable jitter with $25 \%$ correlation. TCP increases its sending rate at RTT timescales, hence for FDPA to promptly respond to rate variations, the estimation interval should be in the order of few RTTs. For this reason we set the estimation interval to 30 ms.

\section{B. Metrics}

We measure the quality of an experiment using two metrics: (i) the aggregate throughput (TPut) normalized over the link capacity, i.e. bounded between 0 and 1, and (ii) the Jain's Fairness Index (JFI) [10]. The JFI is a popular fairness measure defined as:

$$
J F I=\frac{\left(\sum_{n} x_{n}\right)^{2}}{N \cdot \sum_{n} x_{n}^{2}}
$$

where $x_{n}$ is the normalized rate of a user $n$ and $N$ is the total number of users. The normalized rate is defined as $x_{n}=$ MeasuredRate $_{n} /$ FairRate $_{n}$. In our experiments each user is assigned with the same fair share, i.e. Fair Rate ${ }_{n}=$ LinkCapacity/ $N \forall n=1 \ldots N$. The JFI is bounded between 0 and 1 , where 1 is a fair distribution and 0 is a discriminating one. In testing FDPA we aim at maximizing both TPut and JFI.

\section{Results}

Figure 5 shows the results obtained from the experiments. We generate long-lived TCP traffic varying the number of users to 50, 100 and $200,{ }^{1}$ and varying the number of TCP connections per user based on four scenarios: (i) all users open

\footnotetext{
${ }^{1}$ We put a limit to 200 as we noticed that our experimental setup suffers of performance degradation when emulating more users.
} 
1 TCP conn. per user

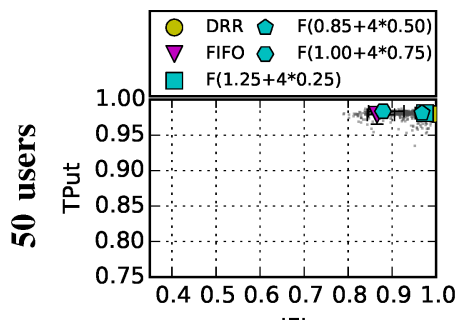

JFI

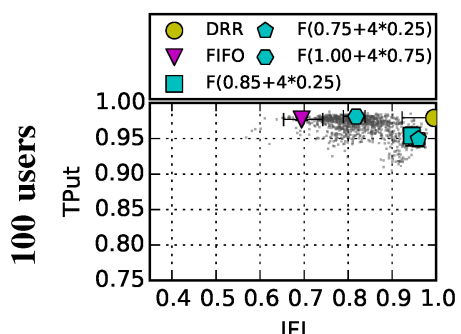

$\mathrm{JFI}$

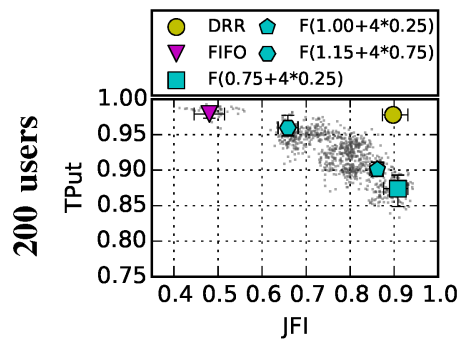

$25 \% 10$ conn.

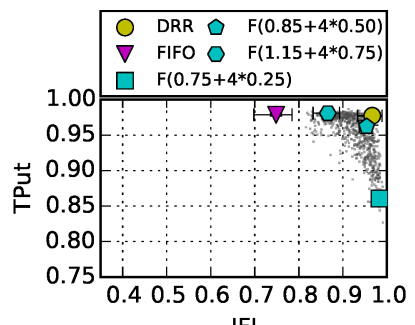

$\mathrm{JFI}$

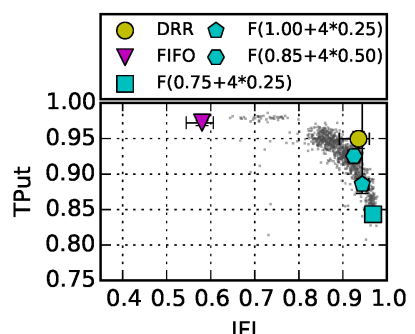

$\mathrm{JFI}$

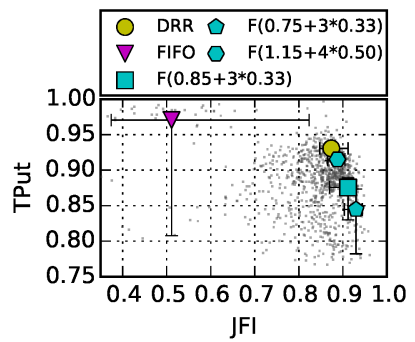

$50 \% 10$ conn.

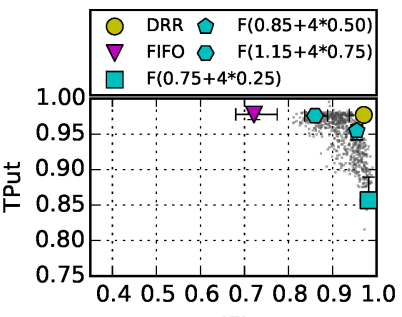

$\mathrm{JFI}$
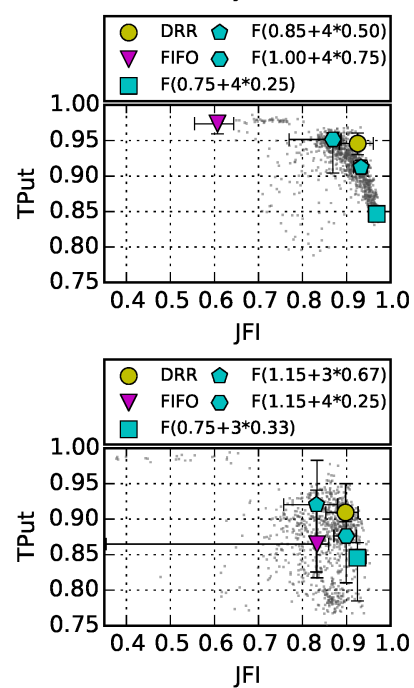

Unif. range 1-10 conn.

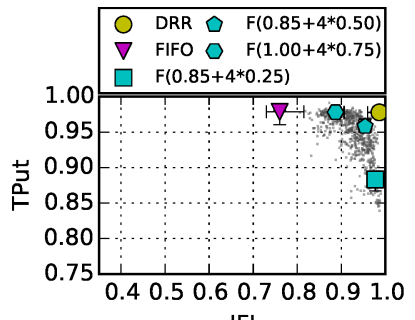

$\mathrm{JFI}$
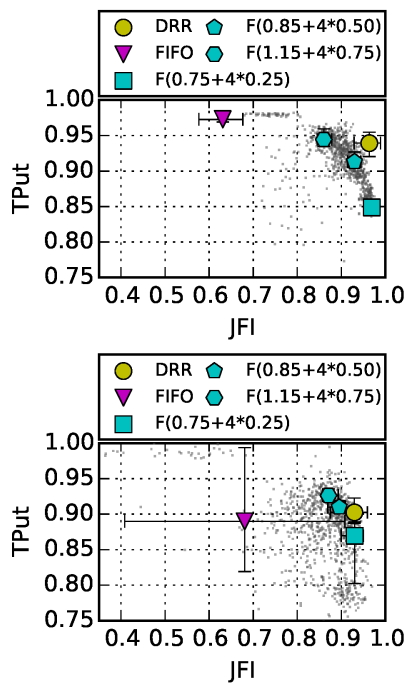

Fig. 5. Experimental results

only one TCP connection, i.e. they all well-behave, (ii) $25 \%$ of the users misbehave by opening 10 parallel TCP connections, while the remaining $75 \%$ only 1 (iii) $50 \%$ of them misbehave, and (iv) the number of connections per user is uniformly distributed between 1 and 10 .

We also vary the number and size of rate bands. We use the following notation to describe an FDPA configuration: $F$ (FirstBand + NumBands $*$ BandSize $)$, where FirstBand is the size of $B_{1}, N u m B a n d s$ is the number of bands following the first one, each one of size BandSize, except for the last one that has infinite size, i.e. up to the link capacity. FirstBand and BandSize are expressed as a proportion of the fair share, e.g. $F(1+4 * 0.5)$ describes a configuration where the first band is exactly the fair share, and the other 4 bands have size half of the latter. We perform experiments with FirstBand $\in\{0.75,0.85,1,1.15,1.25\}$, NumBands $\in$ $\{3,4\}$ and BandSize $\in\{0.25,0.33,0.50,0.67,0.75\}$. For sizing the queues we empirically found that the following rule provides optimal performances: $L_{q}=\min \left(20, B D P / q^{q}\right)$, where $B D P$ is the bandwidth delay product $\overline{R T T} \times$ LinkCapacity. With $\overline{R T T}=5 \mathrm{~ms}$, the sizing for 5 queues is $L_{1}=4166$ MTU-size packets, $L_{2}=1041, L_{3}=154$, $L_{4}=20$, and $L_{5}=20$.

At the server, we collect samples of the average bitrate over a 1-second interval, each second at the same time for all sources, for 50 seconds. We start sampling 30 seconds after starting iperf, allowing all TCP sessions to converge to their average bitrate. For each second, we then compute both the JFI and TPut. In the plots, we show the median of the JFI and TPut samples for each experiment, along with a $80 \%$ confidence interval. For each traffic scenario, we plot only three configurations of FDPA, the one with the best TPut, the one with the best JFI, and the one that maximizes the product of both. We also provide a scatter plot of all JFI and TPut values obtained in all FDPA configurations. This explicitly shows the trade-off between TPut and JFI.

Finally, we compare results with the following cases:

FIFO. All users are served using 1 FIFO queue of size $L=$ $B D P$, e.g. 4166 MTU-size packets with $\overline{R T T}=5 \mathrm{~ms}$. This is our worst case, when fairness is not enforced.

DRR. The switch implements DRR scheduling with per-user queues. We use the tc-drr implementation provided as part of the Linux's tc suite. We use DRR as the best case scenario, however this should be considered as an ideal case. Indeed, while it is still feasible to provide a large number of peruser queues in software, the same does not apply to hardware switches, where an a priori instantiation of hardware resources (memory and logic circuitry) is required for each queue. The reader should remember that the majority of today switching chips provide 10 or less output queues per port [1].

As expected, FDPA holds the promise of enforcing fairness w.r.t. a single FIFO queue in all scenarios, producing results comparable to the ideal case of a DRR scheduler with per-user queues. However, with FDPA fairness comes at the expense of throughput. We observe how configurations of FDPA that 
use narrower bands provide more fairness, between 0.95 and 0.99 in most cases. Unfortunately, these configurations systematically incur in throughput degradation, down to 0.85 in some cases, while for the same scenario DRR achieves almost perfect fairness with throughput comparable to that of a FIFO queue, i.e. optimal around 0.98 , or little less around 0.95 . Vice versa, larger bands improve throughput, at the expense of fairness.

\section{Discussion}

How to improve throughput? Preliminary analysis shows that throughput degradation is mostly caused by packet reordering due to frequent changes in the queue assignment, which confuses the TCP congestion control. A solution to this problem could be that of using a flowlet-based approach [11], in which queue assignments are valid for the whole burst of packets, where bursts are separated by an idle time usually comparable to the RTT. This would decrease the probability of having back-to-back packets sent out from two different queues, and hence packet reordering. Detecting flowlets is a common function implemented by stateful data plane abstractions [7], [12], [20]. We leave exploring such a more advanced design for future work.

Rate estimation. An alternative to average estimators are token bucket-based estimators. The advantage of using token buckets lays in their ability to immediately respond to rate spikes and bursts of packets, while an average estimator might leave enough time to an aggressive user to congest the highest priority queue. We know that the downside of frequent band variations is a higher risk of packet reordering, and preliminary results on our testbed using token buckets show that this is the case. However, we believe that using token buckets along with per-flowlet queue assignment could help in improving both fairness and throughput. We leave this for future work.

How to compute the fair share? We envision an external controller (or switch-internal control plane) that periodically adjusts band sizes by counting the number of active users. In the case of a service provider network, where the number of active users varies slowly, we do not expect that the frequency of the estimation process might be a limit for the scalability of the approach. Indeed, using many priorities helps in absorbing also minor variations of the fair share. How to efficiently implement user estimation is outside the scope of this work, however, we note that a controller could use the same counters instantiated at the switch for the rate estimation process.

\section{Vi. COnclusions}

We introduced FDPA, a design for a packet forwarding pipeline to enforce approximate fair bandwidth sharing. FDPA is based on primitives common in data plane abstractions such as P4 and OpenFlow. Differently from other approaches based on per-packet scheduling, the implementation and time complexity of FDPA does not depend on the maximum number of active users. We performed experiments on a $10 \mathrm{Gbit} / \mathrm{s}$ testbed, results show that performance are close to that of an ideal DRR scheduler with dedicated per-user queues, FDPA instead does not need per-user queues. We identified a tradeoff between fairness and throughput, in which throughput is penalized when configuring FDPA for more fairness. Preliminary analysis show that packet reordering is the cause of such effect. We identified potential solutions to such problem that we leave for future work.

\section{REFERENCES}

[1] Packet buffers. https://people.ucsc.edu/ warner/buffer.html.

[2] G. Bianchi, M. Bonola, A. Capone, and C. Cascone. Openstate: Programming platform-independent stateful openflow applications inside the switch. ACM SIGCOMM CCR 44.2, 2014.

[3] G. Bianchi, M. Bonola, S. Pontarelli, D. Sanvito, A. Capone, and C. Cascone. Open packet processor: a programmable architecture for wire speed platform-independent stateful in-network processing. CoRR, abs/1605.01977, 2016.

[4] N. Bonelli, S. Giordano, and G. Procissi. Network traffic processing with pfq. IEEE JSAC, 34(6), 2016.

[5] P. Bosshart, D. Daly, G. Gibb, M. Izzard, N. McKeown, J. Rexford, C. Schlesinger, D. Talayco, A. Vahdat, G. Varghese, et al. P4: Programming protocol-independent packet processors. ACM SIGCOMM CCR 44.3, 2014.

[6] CAIDA. Analyzing UDP usage in Internet traffic. https://www.caida. org/research/traffic-analysis/tcpudpratio/, 2009.

[7] C. Cascone, L. Pollini, D. Sanvito, and A. Capone. Traffic management applications for stateful sdn data plane. In EWSDN, 2015.

[8] C. Cascone, D. Sanvito, L. Pollini, A. Capone, and B. Sansò. Fast failure detection and recovery in sdn with stateful data plane. International Journal of Network Management, 27(2), 2017.

[9] A. Demers, S. Keshav, and S. Shenker. Analysis and simulation of a fair queueing algorithm. ACM SIGCOMM CCR 19.4, 1989.

[10] R. Jain, D.-M. Chiu, and W. R. Hawe. A quantitative measure of fairness and discrimination for resource allocation in shared computer system. CoRR, cs.NI/9809099, 1998.

[11] S. Kandula, D. Katabi, S. Sinha, and A. Berger. Dynamic load balancing without packet reordering. ACM SIGCOMM CCR 37.2, 2007.

[12] N. Katta, M. Hira, C. Kim, A. Sivaraman, and J. Rexford. HULA: Scalable load balancing using programmable data planes. In ACM SOSR, 2016.

[13] P. E. McKenney. Stochastic fairness queueing. In IEEE INFOCOM, 1990.

[14] N. McKeown, T. Anderson, H. Balakrishnan, G. Parulkar, L. Peterson, J. Rexford, S. Shenker, and J. Turner. Openflow: Enabling innovation in campus networks. ACM SIGCOMM CCR 38.2, 2008.

[15] M. Moshref, A. Bhargava, A. Gupta, M. Yu, and R. Govindan. Flowlevel state transition as a new switch primitive for SDN. In $A C M$ HotSDN, 2014.

[16] J. Nagle. On Packet Switches With Infinite Storage. IETF RFC 970, 1985.

[17] R. Pan, L. Breslau, B. Prabhakar, and S. Shenker. Approximate fairness through differential dropping. ACM SIGCOMM CCR 33.2, 2003.

[18] R. Pan, B. Prabhakar, F. Bonomi, and B. Olsen. Approximate fair bandwidth allocation: A method for simple and flexible traffic management. In IEEE Allerton Conference on Communication, Control, and Computing, 2008.

[19] L. Qiu, Y. Zhang, and S. Keshav. Understanding the performance of many TCP flows. Computer Networks, 37(3-4), 2001.

[20] N. K. Sharma, A. Kaufmann, T. Anderson, A. Krishnamurthy, J. Nelson, and S. Peter. Evaluating the power of flexible packet processing for network resource allocation. In USENIX NSDI, 2017.

[21] M. Shreedhar and G. Varghese. Efficient fair queuing using deficit roundrobin. IEEE/ACM Transactions on networking, 4(3), 1996.

[22] A. Sivaraman, A. Cheung, M. Budiu, C. Kim, M. Alizadeh, H. Balakrishnan, G. Varghese, N. McKeown, and S. Licking. Packet transactions: High-level programming for line-rate switches. In ACM SIGCOMM, 2016.

[23] A. Sivaraman, S. Subramanian, M. Alizadeh, S. Chole, S.-T. Chuang, A. Agrawal, H. Balakrishnan, T. Edsall, S. Katti, and N. McKeown. Programmable packet scheduling at line rate. In ACM SIGCOMM, 2016.

[24] V. Sivaraman, S. Narayana, O. Rottenstreich, S. Muthukrishnan, and J. Rexford. Heavy-hitter detection entirely in the data plane. In $A C M$ SOSR, 2017. 